\title{
Laser-activated drug implant for controlled release to the posterior segment of the eye
}

\author{
Xingyu He, ${ }^{1}$ Zheng Yuan, ${ }^{1}$ Samantha Gaeke, ${ }^{1}$ Winston W.-Y. Kao, ${ }^{2}$ S. Kevin Li, Daniel Miller, ${ }^{2,3}$, \\ Basil Williams, ${ }^{2,4}$ and Yoonjee C. Park ${ }^{1,2, *}$ \\ ${ }^{1}$ Department of Chemical \& Environment Engineering, \\ ${ }^{2}$ Department of Ophthalmology, University of Cincinnati, $\mathrm{OH}$ \\ ${ }^{3}$ Cincinnati Eye Institute, Cincinnati, $\mathrm{OH}$ \\ ${ }^{4}$ Cincinnati Children's Hospital Medical Center, Cincinnati, $\mathrm{OH}$ \\ *Corresponding author: Yoonjee.park@uc.edu
}

\section{Abstract}

The current standard of care for posterior segment eye diseases, such as age-related macular degeneration and diabetic macular edema, is frequent intravitreal injections or sustained-release drug implants. Drug implants have side effects due to the burst release of the drugs, and their release cannot be easily controlled after implantation. Present study attempts to develop a dosage-controllable drug delivery implant which consists of a nanoporous biodegradable PLGA capsule and light-activated liposomes. Controllable drug release from the implant was achieved by using pulsed near-infrared (NIR) laser both in vitro and in vivo. The in vitro drug release kinetics from two different initial dose implants, $1000 \mu \mathrm{g}$ and $500 \mu \mathrm{g}$, was analyzed by fitting zero order and first order kinetics, as well as the Korsmeyer-Peppas and Higuchi models. The $1000 \mu \mathrm{g}$ and $500 \mu \mathrm{g}$ implants fit the first-order and zero-order kinetics model, respectively, the best. The multiple drug releases in the vitreous was determined by in vivo fluorimeter, which was consistent with the in vitro data. The dose released was also clinically relevant. Histology and optical and ultrasound imaging data showed no abnormality in the eyes received implant treatment suggesting that the drug delivery system was safe to the retina. This on-demand dose-controllable drug delivery system could be potentially used for long-term posterior eye disease treatment.

\section{Introduction}

Posterior segment eye diseases such as age-related macular degeneration $(A M D)$, diabetic macular edema (DME) and proliferative vitreoretinopathy (PVR) are serious chronic diseases that may result in vision loss. More than 100 million people around the world are suffering from these chronic posterior eye diseases. ${ }^{1-2}$ The current standard of care for posterior segment eye disease involves frequent repetitive intravitreal injections or sustained-release drug implants. Typically, intravitreal injections are administered monthly or every other month depending on disease and response to treatment. This is due to the relatively short pharmacokinetics of these medications, and there is a relatively short half-life of drugs delivered to the vitreous cavity (within 
hours - days). ${ }^{3}$ However, there is a significant burden to the patient, the patient's family, and the health system because current intravitreal therapies require every 4 to 12 week administration over many years. To resolve the issue, intravitreally injectable sustainedrelease implants have been developed to prolong therapeutic efficacy. ${ }^{4,5}$ Biodegradable implants, such as Ozurdex®), used PLGA (poly lactide-co-glycolic acid) as a drug depot and designed for 6 month-sustained release. The drug release mechanism of Ozurdex implant relies entirely on degradation rate of a PLGA matrix where drug is impregnated. However, most of the drug is released initially after treatment within 1 month due to polymer degradation, ${ }^{6}$ leading to high initial drug concentrations in the vitreous or retina. This high initial dose or burst release of drug from the implant are considered to cause local side effects such as elevated intraocular pressure and cataract formation, etc. ${ }^{7}$

We previously developed a size-exclusive nanoporous biodegradable PLGA capsule as a dosage-controllable drug delivery implant, which was stable and safe in rabbit eyes for 6 months. ${ }^{8}$ In the present study, we combined the capsule with lightactivatable drug-encapsulated liposomes, to create a light-activated dose-controllable implant. Our hypothesis is that light-activatable drug-encapsulated liposomes inside the nanoporous PLGA capsule release drug when irradiated by near-infrared laser irradiation and the drug diffuses through the nanoporous structure to the surrounding media (Figure 1). We focused on drug release from the implant by laser irradiation both in vitro and in vivo to demonstrate feasibility of using the implant as a dose-controllable drug delivery system to the posterior segment of the eye. Drug release kinetics in vitro were analyzed utilizing the implants with two different dosages.

The light-activatable liposomes co-encapsulate methotrexate (MTX) and Cy5 fluorescent dye. MTX is an anti-inflammatory drug, used to treat proliferative vitreoretinopathy (PVR) and non-infectious uveitis. ${ }^{9}$ Cy5 is used to quantitively analyze hydrophilic molecules released by laser trigger in vivo using fluorescence imaging. 


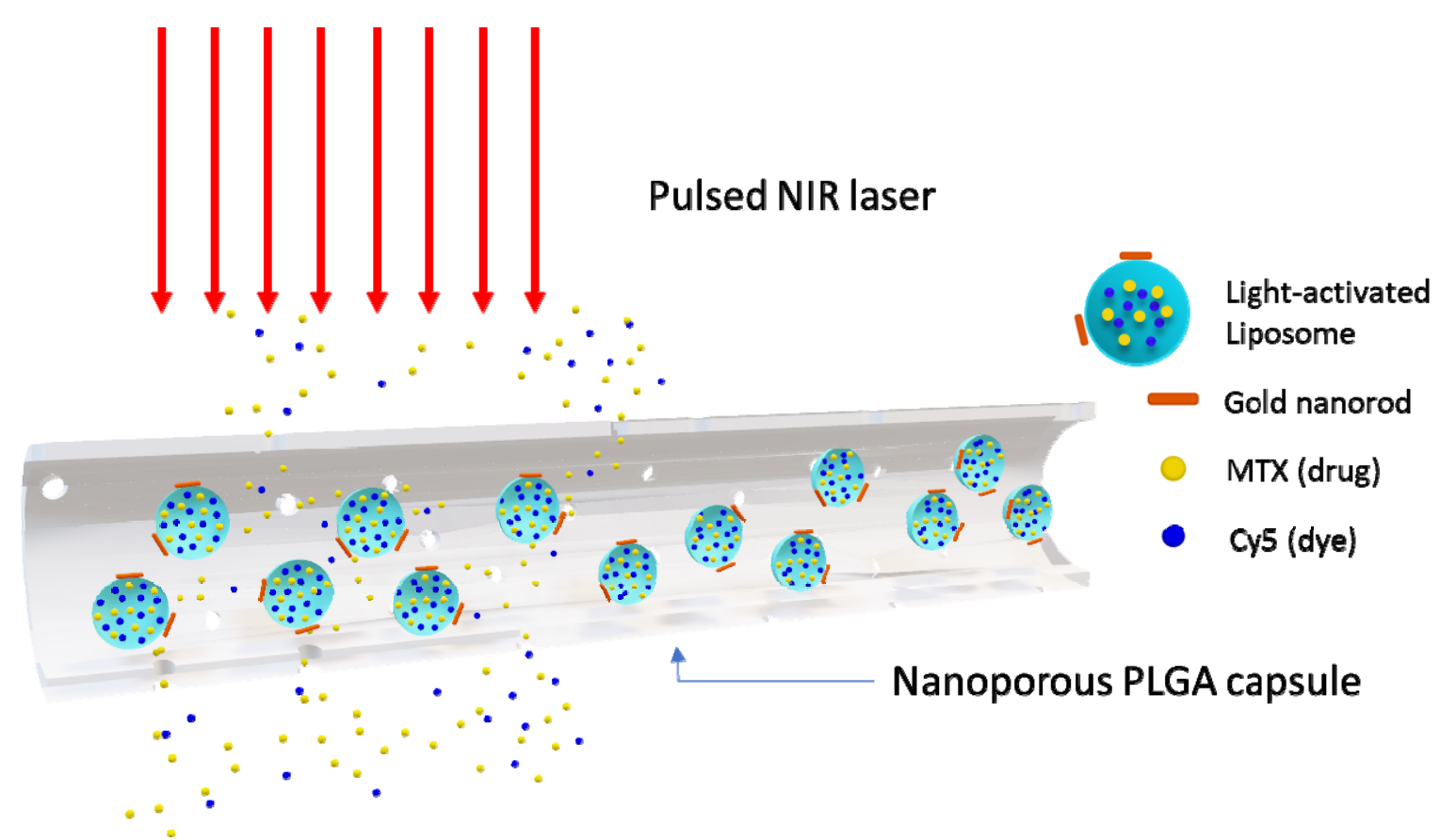

Figure 1.Schematic of light-activated drug implant under pulsed NIR laser irradiation. Drug and dye molecules are released from nanopores of the implant

\section{Materials and methods}

\subsection{Materials}

Poly(lactide-co-glycolide) acid (L/G 90/10, M.W. 200,000) was purchased from PolyScitech, Inc (West Lafayette, IN). Dichloromethane (DCM), chloroform, and polyethylene glycol (PEG, Average MW 3350) were purchased from Fisher Chemical (USA). Stearylamine was purchased from Tokyo Chemical Industry CO.,LTD (Tokyo, Japan). Cholesterol was obtained from MP Biomedicals, LLC (Solon, OH). 1,2distearoyl-sn-glycero-3-phosphoethanolamine-N-[methoxy(polyethylene glycol)-5000 (DSPE-PEG 5000), and 1,2-distearoyl-sn-glycero-3-phosphocholine (DSPC) were purchased from Avanti Polar Lipids, Inc. (Alabaster, AL). Gold-nanorods were purchased from NanoPartz, Inc. (Loveland, CO). Methotrexate (MTX) (Thymoorgan Pharmazie Gmbh, Germany), hydrocortisone and neo-poly-bac ointments were purchased from University of Cincinnati Pharmacy. Sulfo-Cy5 carboxyl acid (Cy5) was purchased from Lumiprobe Co. (Hunt Valley, MD).

\subsection{Synthesis of MTX/Cy5 encapsulated liposomes}

MTX liposomes and MTX/Cy5 co-encapsulated liposomes were synthesized by a modified reverse-phase evaporation (REV) method reported in our previous publication. ${ }^{8}$ MTX liposome was prepared for in vitro drug release test. MTX/Cy5 coencapsulated liposome was used for in vivo drug release test. The MTX or MTX/Cy5 loaded liposome was purified by PD-10 column to remove free drug or dye solution. 50 $\mu \mathrm{L}$ gold nanorod suspension $\left(\sim 4.7 \times 10^{13} / \mathrm{mL}\right.$ ) (NanoPartz, Loveland, CO) was added in 
the purified sample, corresponding to a number ratio of 2:1 for a gold nanorod to a liposome. The mixture was gently shaken for at least $40 \mathrm{~min}$ to have the gold nanorods attach on the surfaces of liposomes via the electrostatic interaction. The attached gold nanorod-coated liposome suspension was concentrated by centrifugation at $6000 \mathrm{rpm}$ for 30 min to achieve a higher drug concentration (accuSpin Micro 17, Fisher Scientific). After centrifugation, the suspension of concentrated drug/dye loaded liposome (approximate $350 \mu \mathrm{L}$ ) was stored at $-20^{\circ} \mathrm{C}$ for $2 \mathrm{~h}$ and then transferred to $-70{ }^{\circ} \mathrm{C}$ for another $2 \mathrm{~h}$ until completely frozen. Then the frozen liposome suspension was lyophilized in a freeze-dryer (FreeZone 2.5, LABCONCO, Kansas City, MO) for $4 \mathrm{~h}$. For $1000 \mu \mathrm{g}$ and $500 \mu \mathrm{g}$ dosage loading, $36.5 \mu \mathrm{L}$ and $73 \mu \mathrm{L}$ of a PBS (phosphate buffer saline) solution, respectively, was added into the lyophilized liposome samples. The rehydrated sample was incubated in room temperature overnight. After stirring the sample with a fine 30-gauge syringe needle was used to manually homogenize the sample for $5 \mathrm{~min}$. Then the gel-like high concentration MTX liposome suspension was centrifuged at $3000 \mathrm{rpm}$ for 3 min to remove any potential bubbles that may occur during the mixing.

The centrifuged samples were diluted 1000 times in PBS. The optical density of diluted samples was measured by UV-vis at $370 \mathrm{~nm}$ which was the absorption peak of MTX. The mixed samples were injected into the PLGA implants. 


\subsection{Fabrication of laser-activated drug implant}

A laser-activated drug release PLGA implant was generated by loading concentrated MTX liposome or MTX/Cy5 liposome in a PLGA nanoporous tube. The PLGA tube was fabricated by mainly following our previous method. ${ }^{8}$ Briefly, PLGA (MW 200,000, L/G 90/10, PolySciTech) was mixed with polyethylene glycol (PEG) (MW 3350, Fisher Chemical) as porogen at 0.1 PEG to PLGA weight ratio in DCM at $50 \mathrm{mg} / \mathrm{ml}$ PLGA in DCM. $800 \mu \mathrm{l}$ of the mixture was transferred to a mold and bath-sonicated for 80 minutes at $10^{\circ} \mathrm{C}$. The PLGA/PEG solution was air dried overnight in a chemical hood at room temperature and became a $\sim 30 \mu \mathrm{m}$ thickness sheet. The PLGA/PEG sheet was rolled using a 22-gauge needle as a template twice to create a double-layer tube. One end of the tube was sealed by a preheated iron at $60^{\circ} \mathrm{C} .4 \mu \mathrm{L}$ of the MTX/Cy5 coencapsulated liposome suspension described above was then injected into the one-end sealed tube using a 30-gauge syringe blunt tip needle. The other end of the tube was sealed in the same manner after sample loading. After sealing, the implant was incubated in $1 \mathrm{~mL}$ PBS at $35^{\circ} \mathrm{C}$ for 24 hours to remove the porogen PEG, resulting in the final product, a nanoporous PLGA capsule implant. Two different liposomal drug doses in the implant, for $1000 \mu \mathrm{g}$ and $500 \mu \mathrm{g}$ implants, the same volume $4 \mathrm{uL}$ but dilution was used. For characterization of the dimension of the PLGA/PEG tube, a digital microscope VHX-2000 (Keyence, IL) was used.

\subsection{In vitro NIR laser irradiation of implant}

A laser-activated implant was placed in a $1.5 \mathrm{~mL}$ test tube with $1 \mathrm{ml} \mathrm{PBS}$ at $35^{\circ} \mathrm{C}$. Each test tube was held horizontally on a petri dish. A pulsed NIR (1064 nm) laser (BrightSolutions, Pavia, Italy) was setup above the test tube. The pulsed NIR laser was irradiated for $15 \mathrm{~min}$ total, $5 \mathrm{~s}$ at 4 different spots $(20 \mathrm{~s})$ with 1 min cooling time between $20 \mathrm{~s}$ irradiations. The released MTX (drug) outside of the implant was quantitively analyzed by using UV-vis spectrometry at absorption wavelength $370 \mathrm{~nm}$. A calibration curve was prepared for MTX concentration in PBS corresponding to its optical density (O.D). PBS solution was replaced after every $24 \mathrm{hr}$. O.D was measured before and after each buffer replacement.

\subsection{Modelling analysis of drug release}

Drug release kinetics was analyzed by fitting cumulative drug release data into following models.

2.5.1. Zero order kinetics

Zero order kinetics is that cumulative drug release is linearly proportional to time, regardless of initial dose in the implant. The equation is

$$
M_{t}=M_{0}+k_{0} t
$$


where $M_{0}$ is the total drug amount in a surrounding medium after reaching "pleatau", $M_{t}$ is the cumulative total amount of drug released in the medium, and $k_{0}$ is a zero-order release kinetic constant.

\subsubsection{First order kinetics}

Drug release rate depends on drug concentration in the drug reservoir, in this case implant. Then,

$$
\frac{d Q}{d t}=-k_{1} Q
$$

where $Q$ is the amount of drug in the implant and $k_{1}$ is a first-order release constant. At time $t=0, Q=Q_{0}$, which is the total amount of drug released from the implant until reaching plateau. Then,

$$
\ln \frac{Q_{t}}{Q_{0}}=-k_{1} t
$$

and $Q_{t}=Q_{0}-M_{t}$.

\subsubsection{Korsmeyer-Peppas (KP) model}

The generic equation for the Korsmeyer-Peppas model is

$$
F=\frac{M_{t}}{M_{0}}=k_{K P} t^{n}
$$

where $F$ is fraction of drug released at time $t, M_{t}$ is drug release from implant at time, $M_{0}$ is the total drug release from implant until reaching "plateau". $k_{K P}$ is a KorsmeyerPeppas kinetic constant, and $n$ is diffusion or release exponent, which will be determined by fitting. $n$ suggests type of drug release mechanism as shown in Table 1.

Table 1. Indication of $n$ value for Korsmeyer-Peppas equation..$^{10-11}$

\begin{tabular}{|c|c|}
\hline $\mathbf{n}$ & Indication \\
\hline$<0.45$ & Quasi fickian \\
\hline 0.5 & Fickian diffusion \\
\hline $0.55<\mathrm{n}<1$ & $\begin{array}{c}\text { Anomalous diffusion } \\
\text { or Non-fickian diffusion }\end{array}$ \\
\hline $\mathrm{n}=1$ & $\begin{array}{c}\text { Case II diffusion } \\
\text { Or Zero-order release }\end{array}$ \\
\hline $\mathrm{n}>1$ & Non-fickian super case II \\
\hline
\end{tabular}

The Korsmeyer-Peppas model is applicable to assess the mechanism of the first $60 \%$ of drug release taking place from swellable polymeric devices. ${ }^{11}$

2.5.4. Higuchi model 
Drug release mechanism primarily caused by diffusion process can be anticipated by fitting the drug release data to the Higuchi release equation as the following. ${ }^{12}$

$$
\frac{M_{t}}{M_{0}}=k_{H} t^{\frac{1}{2}}
$$

where $M_{t}$ is drug release from implant at time, $M_{0}$ is the total drug release from implant by laser irradiation and $k_{H}$ is Higuchi constant. It describes drug release as a diffusion process based on Fick's law.

\subsubsection{Statistical analysis of drug release kinetics}

Statistical comparisons between release kinetics $\left(k_{0}, k_{1}, k_{K P}, k_{H}\right)$ of $1000 \mu \mathrm{g}$ implant and $500 \mu \mathrm{g}$ implant under zero order, first order, KP equation and Higuchi model (with and without first 24 hours data) were performed by Single Factor ANOVA, and p-value $<0.05$ was considered statistically significantly different $(n=3)$.

2.6. Fluorescence calibration of remaining MTX in implants Implants loaded with 700, 800, 900, and $1000 \mu \mathrm{g}$ MTX dosage of MTX/Cy5 coencapsulated liposome suspension were characterized by an in vivo fluorescent microscope (Micron IV, Phoenix Technology Group LLC., CA). The fluorescent intensity of Cy5 dye in the implant was quantified by a mean gray value in ImageJ $(\mathrm{NIH})$. A calibration curve was generated based on the mean gray values in the implant corresponding to the dosage loaded.

\subsection{In vivo drug release from implant}

All animal studies were performed at the Laboratory Animal Medical Services and approved by the IACUC at the University of Cincinnati. The drug release of our laseractivated implant was determined in New Zealand White rabbits $(\sim 2 \mathrm{~kg})$. Before injection, the implant was sterilized by ultraviolet (UV) for 30 minutes and inserted into a sterilized 17-gauge needle. The implant was injected into the vitreous at the pars plana through the sclera around 3-5 $\mathrm{mm}$ away from the limbus performed by a surgeon. Untreated eyes served as the control group.

The implant in the rabbit eye was examined by optical/fluorescence imaging (Micron IV), ultrasound imaging (Terason Ultrasound, Burlington, MA) and fundus imaging (28D fundus lens, Volk Optical, Mentor, $\mathrm{OH}$ ). A guide beam was used to aim the center of injected implant. After alignment, a near-infrared pulsed laser $\left(1064 \mathrm{~nm}, 1.1 \mathrm{~W} / \mathrm{cm}^{2}\right)$ was replaced with the guide beam. Then the implant was irradiated for $20 \mathrm{~s}$ (5 s x 4 different spots along the implant) and halted for 3 min for cool-down. After cooling, irradiation was performed again in the same manner until $10 \mathrm{~min}$ total. After the laser irradiation, optical imaging, ultrasound imaging and fluorescence imaging were used to characterize the implant and surrounding area in the vitreous. The lens of microscope was contacted on the cornea of the rabbit with an eye gel (Genteal Tears, Fort Worth, TX). The amount of drug released in vivo was quantified by analyzing the fluorescent intensity of the implant using a mean gray value in ImageJ. Based on the calibration 
curve obtained in Section 2.6, drug concentration in the implant remained after irradiation was determined. The control eyes did not receive laser irradiation and were imaged in the same manner.

\subsection{Tissue processing and histological examination}

Dissection of the eyes was immediately performed in a sterile biosafety fume hood after eye enucleation. A short incision was carefully made near the limbus using a sharp razor blade to separate the anterior and posterior segments of the eye. Gross images of the posterior segment were taken to show the original position and appearance of the implant. About a quadrant of the posterior segment which was closest to the implant was carefully separated by fine scissors and a razor blade for histological examination. The control eye remained unopened. The isolated segment for the implant+laser group and the control eye were fixed in Davidson's fixative solution (comprised of 3 parts of $95 \%$ ethanol, 2 parts of $37 \%$ formaldehyde, 3 parts of distilled water and 1 part of glacial acetic acid) for 24 hours at room temperature. Then they were transferred to freshly prepared $4 \%$ paraformaldehyde solution at room temperature until dehydration. The tissue was dehydrated in graded ethanol (50\%-100\%), cleared in CitriSolv, and subsequently embedded in paraffin. Sequential sections with $8 \mu \mathrm{m}$ thickness were cut, collected and stained with hematoxylin and eosin (H\&E). The histological images were taken by a Nikon Eclipse E800 microscope (Nikon, Tokyo, Japan).

\section{Results}

\subsection{Characterization of laser-activated drug implant}

The PLGA tube prepared from the PLGA/PEG sheet showed the thickness (d), $59.0 \pm$ $7.2 \mu \mathrm{m}$, and the inner radius $(\mathrm{R}), 293.2 \pm 2.3 \mu \mathrm{m}$ (Figure 2, middle). The implant where the lumen was filled with $4 \mu \mathrm{L}$ of rehydrated MTX/Cy5 liposomes was inserted in a syringe needle for intravitreal injection (Figure 2, right). The concentrations of liposomal MTX in the implant for $1000 \mu \mathrm{g}$ and $500 \mu \mathrm{g}$ implants were $245 \pm 9.9 \mathrm{mg} / \mathrm{ml}$ and $119.5 \pm$ $13.4 \mathrm{mg} / \mathrm{ml}$, respectively, determined by UV-Vis spectroscopy.
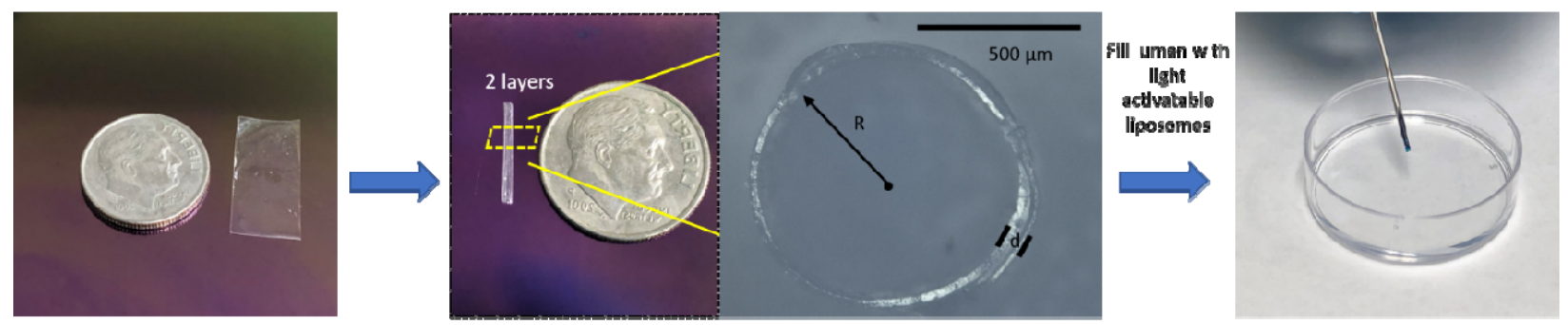

Figure 2.Process of preparation of dye/drug co-encapsulated liposome loaded in a two-layers PLGA implant.

3.2 In vitro drug release of implant after laser irradiations 
Cumulative amounts of MTX released from the $1000 \mu \mathrm{g}$ and $500 \mu \mathrm{g}$ implants after the laser irradiation trigger are demonstrated in Figure 3. Implant initially loaded with 1000 $\mu \mathrm{g}$ implant showed a rapid release within first $24 \mathrm{hr}$. The release kinetics slope decreased after $24 \mathrm{hr}$ and it reached almost a plateau after $168 \mathrm{hr}$. For $500 \mu \mathrm{g}$ implant, the profile was relatively linear with time. The plateau showed up at 192 hours. Overall, $\sim 50 \mu \mathrm{g}$ and $\sim 25 \mu \mathrm{g}$ of MTX were released from the $1000 \mu \mathrm{g}$ and $500 \mu \mathrm{g}$, respectively, per irradiation. The results suggest that the "total" dose released by laser was proportional to the initial dose packed in the implant, in other words, the drug concentration.

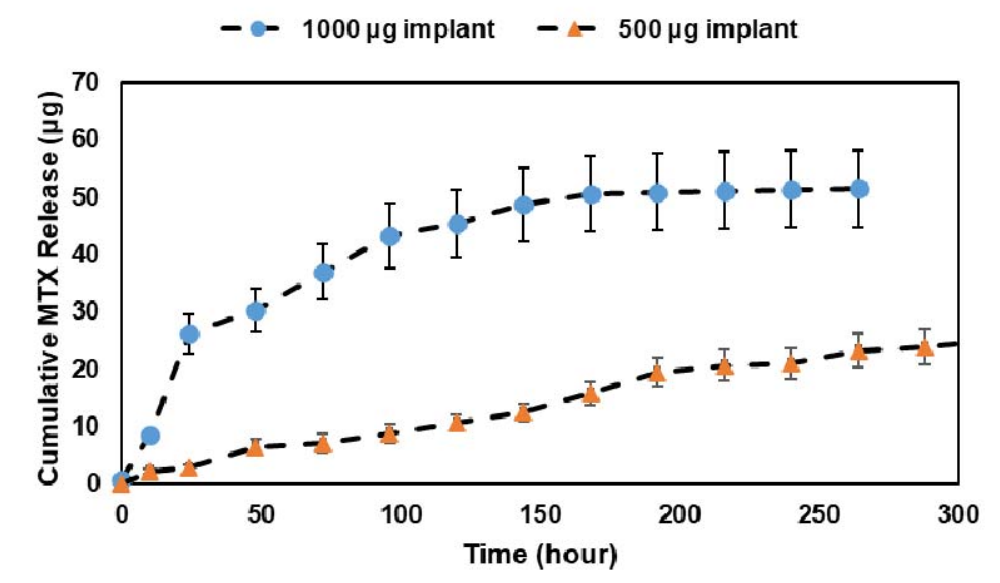

Figure 3. Cumulative amount of MTX released from implants after irradiation with two different total doses as in liposomal form packed in the implant: 1000 $\mu \mathrm{g}$ (b/ue circles) and $500 \mu \mathrm{g}$ (orange triangles).

\subsection{Drug release kinetics models}

Cumulative MTX release data were analyzed by fitting into four drug release kinetics models, including zero-order, first-order, Korsmeyer-Peppas, and Higuchi models (Figure 4). For the zero-order fittings, the $500 \mu \mathrm{g}$ implants showed a higher regression coefficient $\left(R^{2}=0.99\right)$ than the $1000 \mu g\left(R^{2}=0.90\right)$. (Figure 4(A), Table 2). In fact, the 500 $\mu \mathrm{g}$ kinetic curve fitted the zero-order kinetics the best among the four models. The zeroorder rate constants were $k_{0}=0.0044$ and $k_{0}=0.0036$ for $1000 \mu \mathrm{g}$ and $500 \mu \mathrm{g}$, respectively, indicating that the $1000 \mu \mathrm{g}$ implants released the drug through the capsule membrane faster than the $500 \mu \mathrm{g}$. Because of the fast increase of drug release within $24 \mathrm{hr}$ for the $1000 \mu \mathrm{g}$ implants, we have fitted the data excluding data points before 24 $\mathrm{hr}$. Regardless, the $\mathrm{R}^{2}$ values did not improve in the zero-order kinetics for the $1000 \mu \mathrm{g}$ $\left(R^{2}=0.82\right)$. For the first-order fitting, the $1000 \mu \mathrm{g}$ drug release kinetic curve fitted the best among the four models $\left(R^{2}=0.99\right)$, suggesting that the rate of drug release from $1000 \mu \mathrm{g}$ implants was proportional to the drug concentration released by laser in the implant (Figure 4(B), Table 2). The $R^{2}$ for the $500 \mu \mathrm{g}$ implants in the first-order kinetic model was 0.95 . The first-order kinetic rate constants for $1000 \mu \mathrm{g}$ and $500 \mu \mathrm{g}$ were $k_{1}$ 
$=0.022$ and $k_{1}=0.0089$, respectively, indicating that the drug release rate of the $1000 \mu \mathrm{g}$ was much faster than the $500 \mu \mathrm{g}$.

For KP model in Figure $4(\mathrm{C})$, since $\log (\mathrm{t})$ at $\mathrm{t}=0$ cannot be plotted, the first data point started from $12 \mathrm{hr}$. The $\mathrm{n}$ values from KP model fittings for $1000 \mu \mathrm{g}$ and $500 \mu \mathrm{g}$ were 0.47 and 0.78 , respectively, suggesting that they showed different diffusion mechanisms according to Table $1 . \mathrm{n}=0.47$ for the $1000 \mu \mathrm{g}$ suggested that the drug release from 1000 $\mu \mathrm{g}$ followed Fickian diffusion although the $R^{2}$ value was low $\left(R^{2}=0.88\right) . n=0.78$ for the $500 \mu \mathrm{g}$ suggested it followed non-Fickian diffusion with relatively high $\mathrm{R}^{2}$ values, at $\mathrm{R}^{2}$ $=0.97$ and $R^{2}=0.99$, including data points before $24 \mathrm{hr}$ and excluding $24 \mathrm{hr}$, respectively. However, the Higuchi model fitting in Figure 4(D) depicts Fickian diffusion, and $R^{2}$ values for both $1000 \mu \mathrm{g}$ and $500 \mu \mathrm{g}$ were high at 0.98 and 0.98 , respectively, indicative of Fickian diffusion-controlled release. The slopes from Higuchi model showed that $k_{H}=0.072$ and $k_{H}=0.052$ for $1000 \mu \mathrm{g}$ and $500 \mu \mathrm{g}$, respectively, suggesting drug release from the $1000 \mu \mathrm{g}$ was faster than the $500 \mu \mathrm{g}$.

The KP model originally describes different diffusion mechanisms stem from changes in polymer state or morphology upon hydration. ${ }^{10}$ However, our implant was incubated and equilibrated in an aqueous solvent (PBS) before irradiation. It is unlikely polymer morphology changes from glass to rubber phase due to "swelling" during the drug release. Instead, as the KP model suggests diffusion in glassy polymers may be either Fickian or non-Fickian, depending on the relative rates of chain relaxation and diffusion, and on the activity of water in the polymer. PLGA is glassy compared to the polymers that Korsmeyer and Peppas used, such as PVA hydrogel. Overall, both KP model and Higuchi model suggest drug release mechanism follows diffusion through solid structure.

To confirm the differences in the release of MTX from the $1000 \mu \mathrm{g}$ and $500 \mu \mathrm{g}$ implants tested, statistical analysis by Single Factor ANOVA was performed (Table 2). The pvalues of most groups were less than 0.05 except zero-order from $0 \mathrm{hr}$, KP model from $0 \mathrm{hr}$, and Higuchi model from $24 \mathrm{hr}$, indicating the rate constants and $\mathrm{n}$ values were significantly different between the $1000 \mu \mathrm{g}$ and the 500 . The $\mathrm{p}$-value for the zero-order rate constant $k_{0}$ from $0 \mathrm{hr}$ was 0.32 . The $k_{0}$ was sensitive to the final cumulative drug release amount. Relatively large variations in the $1000 \mu \mathrm{gg}$ group \%cumulative release, from $37 \%$ to $62 \%$, may have affected the large $p$-value. The $p$-value for the KP model from $0 \mathrm{hr}$ was 0.091 and the big error bars in the initial data points of the $500 \mu \mathrm{g}$ group indicate that the variation could have caused the large p-values. Overall, the statistical analysis implies that each model emphasizes different release mechanisms. 

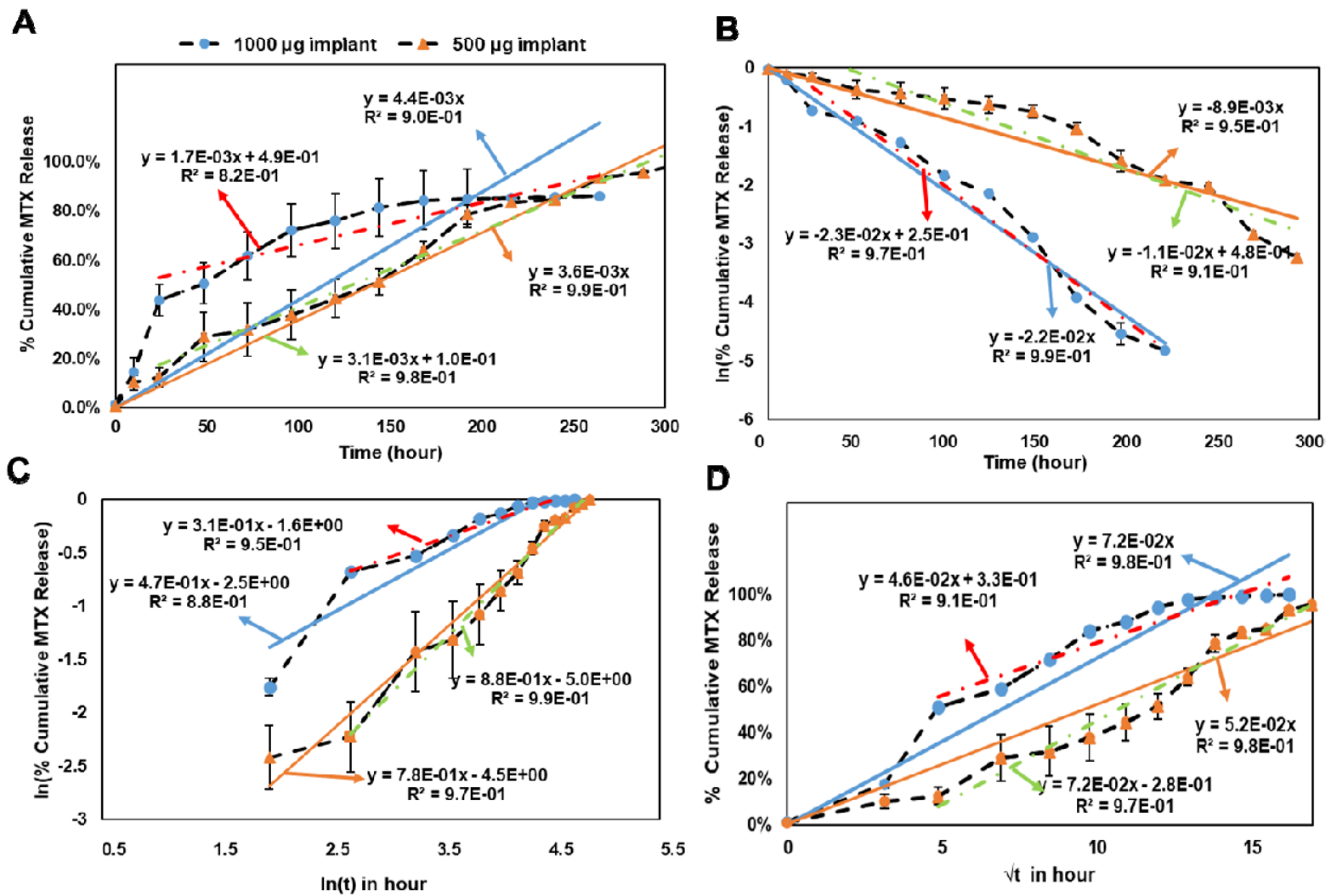

Figure 4. Fitting results of cumulative MTX release kinetics by laser irradiation according to (A) zero-order (B) first-order (C) Korsmeyer-Peppas and (D) Higuchi models. Data: $1000 \mu \mathrm{g}$ (blue circles) and $500 \mu \mathrm{g}$ (orange triangles). Trend lines: $1000 \mu \mathrm{g}$ from $0 \mathrm{hr}$ (blue solid), $1000 \mu \mathrm{g}$ from $24 \mathrm{hr}$ (red dash-dot), $500 \mu \mathrm{g}$ from $\mathrm{O} \mathrm{hr}$ (orange solid), and $500 \mu \mathrm{g} \mathrm{from} 24 \mathrm{hr}$ (green dash-dot).

Table 2. p-values of drug release kinetics for $1000 \mu \mathrm{g}$ and $500 \mu \mathrm{g}$ implants.

\begin{tabular}{|c|c|c|c|c|c|c|}
\hline Fitting models & $\begin{array}{c}k \\
\text { (from } 0 \mathrm{hr}\end{array}$ & $\begin{array}{c}\mathrm{R}^{2} \\
\text { (from } 0 \mathrm{hr} \text { ) }\end{array}$ & $\begin{array}{c}k \\
\text { (from } 24 \mathrm{hr} \text { ) }\end{array}$ & $\begin{array}{c}\mathrm{R}^{2} \\
\text { (from } 24 \mathrm{hr} \text { ) }\end{array}$ & $\begin{array}{l}\mathrm{p} \text {-values } \\
\text { (from } 0 \mathrm{hr} \text { ) }\end{array}$ & $\begin{array}{c}p \text {-values } \\
\text { (from } 24 \text { hr) }\end{array}$ \\
\hline $\begin{array}{l}\text { Zero order } \\
(1000 \mu \mathrm{g})\end{array}$ & $k_{0}=0.0044$ & 0.90 & $k_{0}=0.0017$ & 0.82 & \multirow{2}{*}{0.32} & \multirow{2}{*}{0.048} \\
\hline $\begin{array}{l}\text { Zero order } \\
(500 \mu \mathrm{g})\end{array}$ & $k_{0}=0.0036$ & 0.99 & $k_{0}=0.0031$ & 0.98 & & \\
\hline $\begin{array}{l}\text { First order } \\
(1000 \mu \mathrm{g})\end{array}$ & $k_{1}=-0.022$ & 0.99 & $k_{1}=-0.023$ & 0.97 & \multirow{2}{*}{0.00060} & \multirow{2}{*}{0.0016} \\
\hline $\begin{array}{l}\text { First order } \\
(500 \mu \mathrm{g})\end{array}$ & $k_{1}=-0.0089$ & 0.95 & $k_{1}=-0.011$ & 0.91 & & \\
\hline $\begin{array}{l}\text { KP model } \\
(1000 \mu \mathrm{g})\end{array}$ & $\begin{array}{l}n_{K P}=0.47 \\
k_{K P}=0.085\end{array}$ & 0.88 & $\begin{array}{c}n_{K P}=0.31 \\
k_{K P}=0.2019\end{array}$ & 0.95 & \multirow{2}{*}{$\begin{array}{l}0.091\left(n_{K P}\right) \\
0.024\left(k_{K P}\right)\end{array}$} & \multirow{2}{*}{$\begin{array}{c}0.045\left(n_{K P}\right) \\
0.0024\left(k_{K P}\right)\end{array}$} \\
\hline $\begin{array}{c}\text { KP model } \\
(500 \mu \mathrm{g})\end{array}$ & $\begin{array}{l}n_{K P}=0.78 \\
k_{K P}=0.011\end{array}$ & 0.97 & $\begin{array}{c}n_{K P}=0.88 \\
k_{K P}=0.0067\end{array}$ & 0.99 & & \\
\hline $\begin{array}{l}\text { Higuchi model } \\
(1000 \mu \mathrm{g})\end{array}$ & $k_{H}=0.072$ & 0.98 & 0.046 & 0.91 & \multirow{2}{*}{0.0044} & \multirow{2}{*}{0.066} \\
\hline $\begin{array}{l}\text { Higuchi model } \\
(500 \mu \mathrm{g})\end{array}$ & $k_{H}=0.052$ & 0.98 & 0.072 & 0.97 & & \\
\hline
\end{tabular}




\subsection{In vivo drug/dye release after laser irradiation}

The in vivo study involved intravitreal injection of the implant into the vitreous cavity of rabbits followed by weekly irradiation of the implant with pulsed laser. In Figure 5, the long cylindrical shape of the implant in the vitreous was observed by ultrasound and optical images for the implant + laser group, as opposed to the control group. The ultrasound images show sagittal views that includes the lens on top of the image and the vitreous and the retina. The implant was positioned diagonally following the direction of injection and the position or location did not change over 21 days. The optical (OP) and ultrasound (UL) images in Figure 5 were taken before laser irradiation. Except the Day 0 image, Days 7, 14, and 21 images showed a smudge of dye near the implant, indicative of dye/drug released from the previous week. 


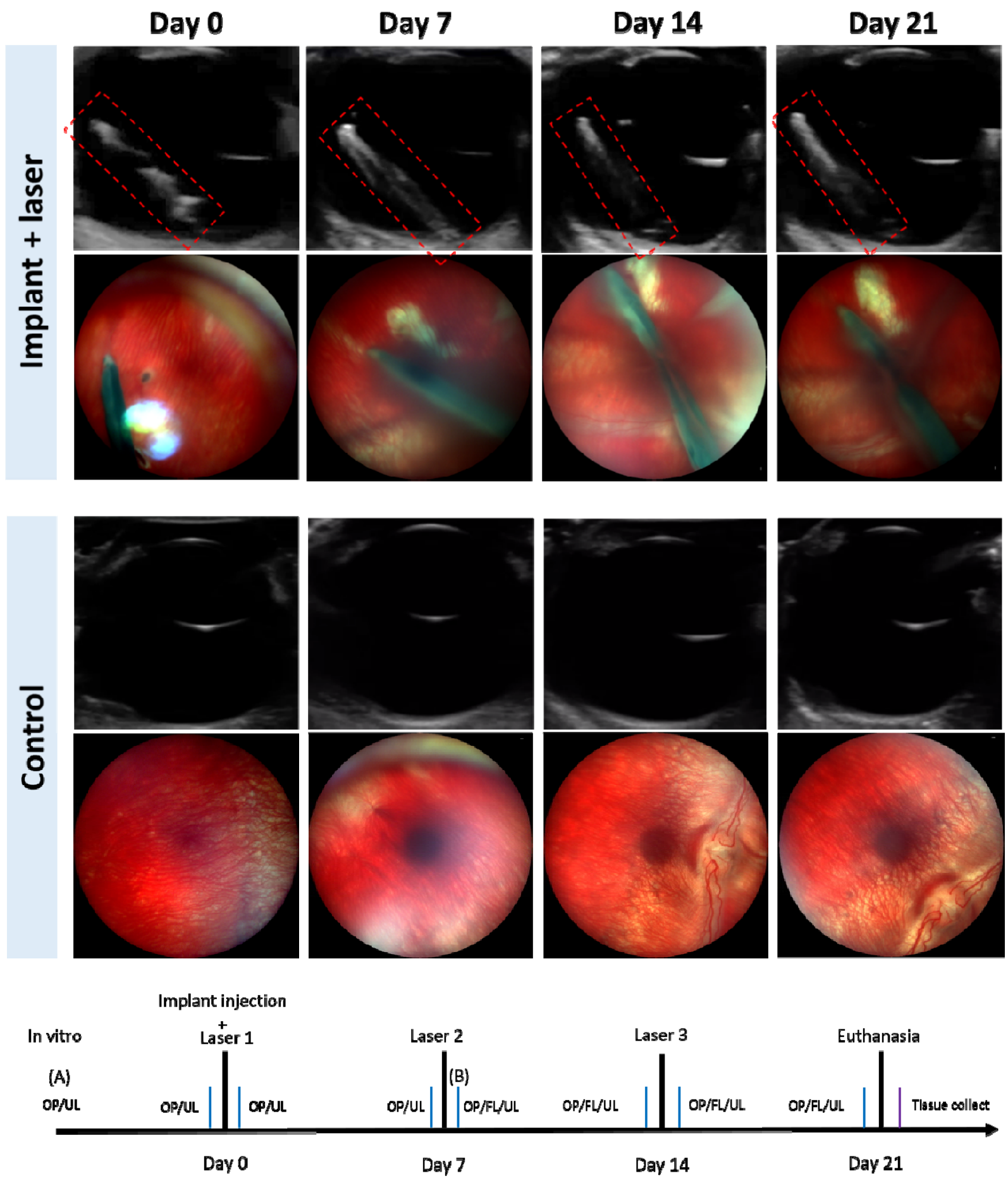

Figure 5. Ultrasound and optical images of the implant-injected eye showing the retina and the implant, and the control eye on Day 0, Day 7, Day 14 and Day 21, respectively. The laser was irradiated at Days 0, 7, and 14 for the implant group.

Based on the in vivo fluorescence images (FL) in Figure 6, the amount of the drug/dye released by laser was estimated. The fluorescence images of the $1000 \mu \mathrm{g}$ implant 
before first irradiation and after second irradiations are demonstrated in Figure $6(\mathrm{~A})$ and $(B)$, respectively. The mean (average) gray values of the whole implant analyzed by ImageJ were 64.1 and 44.6 for $(A)$ and (B), respectively, indicating the dye/drug was released by laser irradiation in vivo. On the other hand, the mean gray values of the whole vitreous increased from 0 to 33.9. Based on the mean gray values in the implant and the calibration curve between the mean gray value in the implant and MTX drug amount in the implant (Supplementary S2), the amounts of MTX in the implant for (A) and $(\mathrm{B})$ were $1000 \mu \mathrm{g}$ and $898.7 \mu \mathrm{g}$, respectively. This suggests that about $100 \mu \mathrm{g}$ of MTX was released by two irradiations. The results match the in vitro results, where $1000 \mu \mathrm{g}$ implants released $\sim 50 \mu \mathrm{g}$ per irradiation. The mean gray values in the center of implant significantly decreased from 84 to 42 , indicating that the dye/drug were released from the area where laser irradiated.

(A)

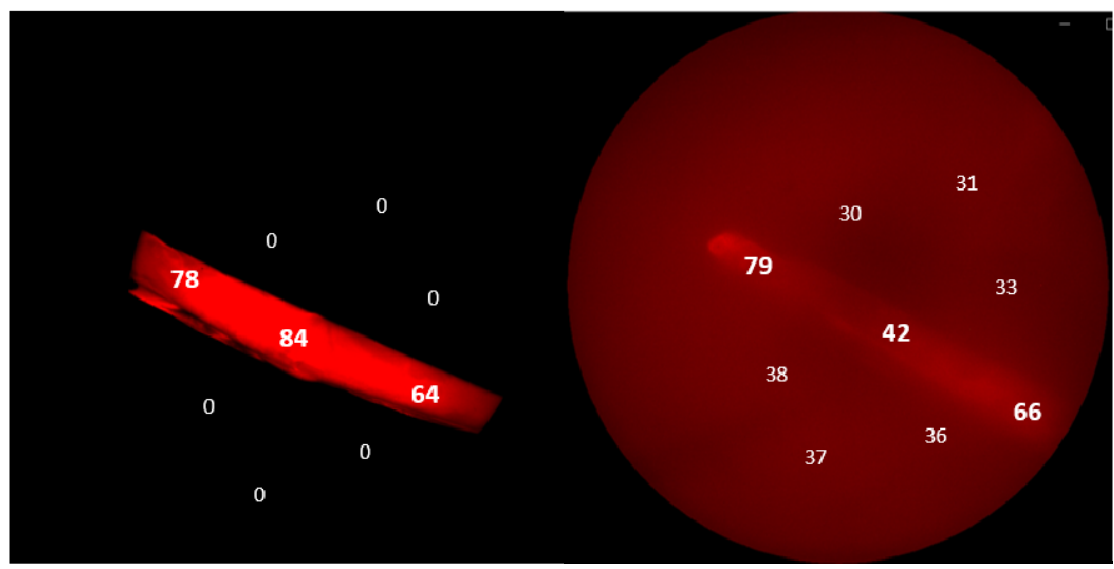

\begin{tabular}{|c|c|c|}
\hline Image & $(\mathrm{A})$ & $(\mathrm{B})$ \\
\hline $\begin{array}{c}\text { Mean gray value in } \\
\text { implant }\end{array}$ & 64.1 & 44.6 \\
\hline $\begin{array}{c}\text { Mean gray value in the } \\
\text { vitreous }\end{array}$ & 0 & 33.9 \\
\hline $\begin{array}{c}\text { Estimated MTX remained } \\
\text { in implant }(\mu \mathrm{g})\end{array}$ & 1000 & 898.7 \\
\hline
\end{tabular}

Figure 6. Mean gray values of fluorescent images of $(A)$ before laser and (B) after two lasers in vivo.

\subsection{In vivo safety of the light-activated drug delivery implant}

Based upon the ultrasound and optical images in Figure 5, the implant + laser group did not show any adverse effect on the retina over the 21-day period. The retina margin looked clear and did not change over time in the ultrasound images. Also, in the optical images, abnormal vessels or structures in the retina were not observed. The location and the shape of the implant did not change according to the results of ultrasound and optical imaging. 
Figure 7(A) presents the gross picture of the eye cup from the implant+laser group after 21 days and 3 irradiations, showing the intactness of the implant. No obvious damage was observed on the outer surface of the implant. The histological sections from the implant+laser group showed the retinal layers remained their integrity, including nerve fiber layer, inner nuclear layer, outer nuclear layer, retinal pigment epithelium (RPE) and choroid, suggesting minimal toxicity from the implant (Figure 7B). In addition, multinucleated giant cells, which are a typical indicator of foreign body response ${ }^{13}$, were not found in the histological sections. The detachment observed of the retina in the implant eye was due to tissue preparation process, compared to the control eye which was fixed intact without a cut-open for tissue preparation (Figure $8 \mathrm{C}$ ). Both the optical imaging and histology data showed no sign of laser damages, which are often characterized by RPE and choroidal circular pigmentation, and loss of nuclei in the outer nuclear layer (ONL). ${ }^{14} 15$
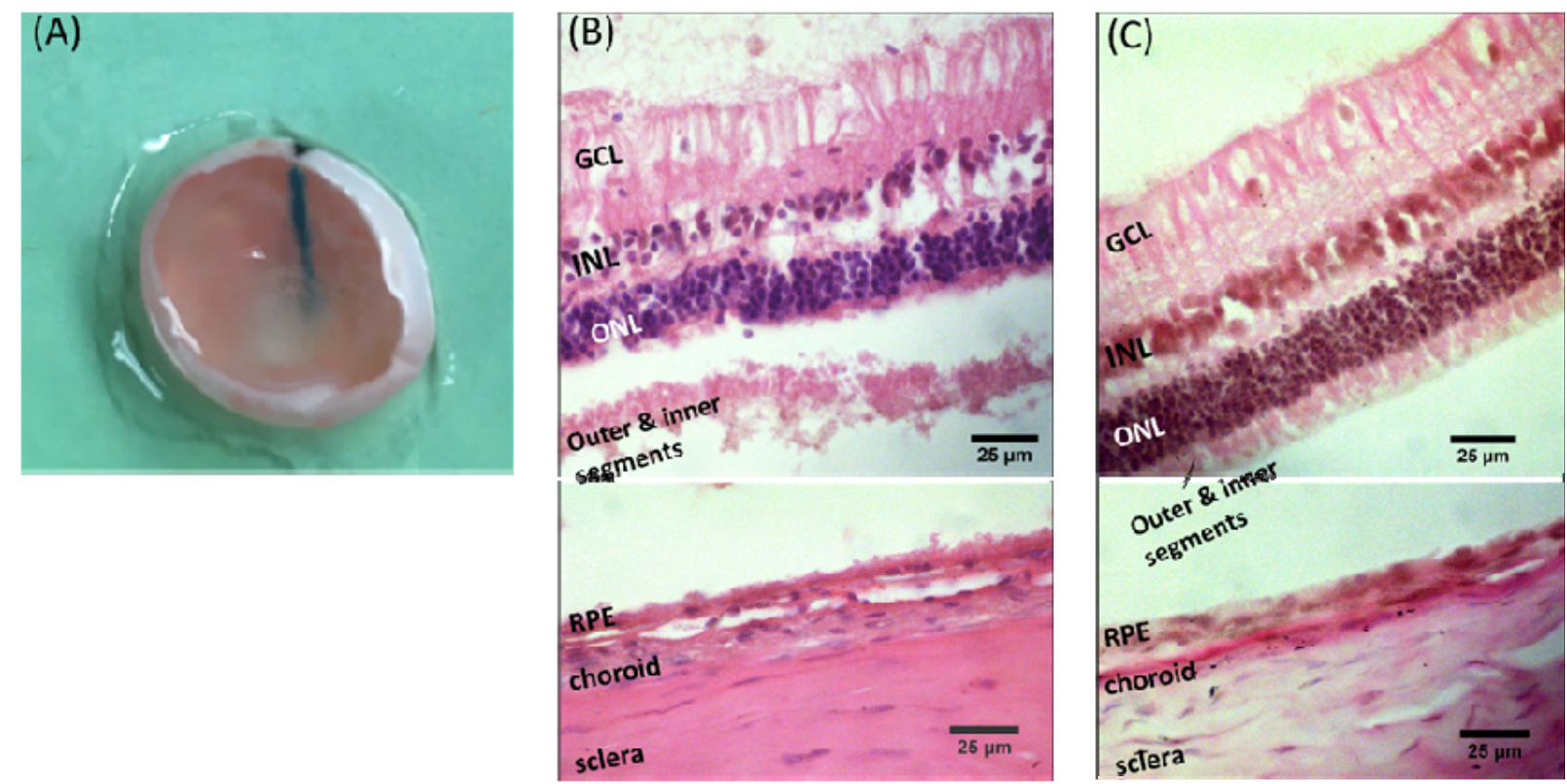

Figure 7. (A) Gross picture of the implant + laser rabbit eye after removing the lens and cornea. Black ink was used to mark the location of implant from outside the sclera. Representative histological images showing the retinal layers with H\&E staining of (B) the implant+laser eye and (C) the control eye. GCL: ganglion cell layer; INL: inner nuclear layer; ONL: outer nuclear layer; $R P E$ : retinal pigment epithelium.

\section{Discussion}

Previously, we have tested the long-term stability of the implant for 6 months in vivo and in vitro and the safety in the rabbit eyes. ${ }^{8}$ There were no signs of significant leakage of drug from the implant for 4 months post injection and no cytotoxicity or immune responses were observed after 6 months implantation. In this study, we focused on 
irradiation induced drug release and achieved a clinically relevant vitreous cavity dose in vivo and in vitro. First, we utilized a lyophilization technique to increase the initial dose of the liposomal drug in the implant compared to our previous study to make the dose relevant to clinical doses. One clinical study showed that $100 \sim 200 \mu \mathrm{g}$ of MTX per injection biweekly was therapeutically effective to treat proliferative vitreoretinopathy. ${ }^{16}$ Second, the drug dose released per irradiation was also comparable to the clinical dose. Although the total dose of $\sim 50 \mu \mathrm{g}$ released from the implant after the laser irradiation trigger in this study does not reach the normal clinical dose of $100 \sim 200 \mu \mathrm{g}$ MTX, the implant provides higher drug concentration in the vitreous over several days than conventional intravitreal injection. This is because the amount of drug in the vitreous after intravitreal injection would significantly drop within a day because of the half-life of free MTX molecules in the vitreous, $14.3 \mathrm{hr}^{17}$ In other words, a half of $100 \mu \mathrm{g}, 50 \mu \mathrm{g}$, is cleared out from the vitreous in less than a day. On the other hand, the light-activated implant continuously released the $50 \mu \mathrm{g}$ over 10 days after laser irradiation, keeping a dose of $\sim 5 \mu \mathrm{g}$ daily. This new delivery mechanism is beneficial for long-term drug delivery. We have also shown repetitive multiple laser-triggered releases in vivo. After 2 irradiations, $100 \mu \mathrm{g}$ was released from the implant, and theoretically the $1000 \mu \mathrm{g}$ implant has the capacity for 20 times laser triggers.

The "total" drug release by one laser irradiation was proportional to the total dose packed in the implant: $\sim 50 \mu \mathrm{g}$ from $1000 \mu \mathrm{g}$, and $\sim 25 \mu \mathrm{g}$ from $500 \mu \mathrm{g}$ implants. The $1000 \mu \mathrm{g}$ has double the concentration of liposomal drug compared to the $500 \mu \mathrm{g}$ implant. Thus, it is likely the higher "total" drug release from the higher dose implants was because of more liposomal drug hit by the laser. In addition, the higher "total" dose released by laser irradiation induced distinct drug release kinetics: the $1000 \mu \mathrm{g}$ and the $500 \mu \mathrm{g}$, respectively, followed first-order kinetics and zero-order kinetics the best. The results imply that the high "total" dose generated a concentration gradient across the implant capsule membrane and the concentration gradient drives the diffusion faster than the low dose. On the other hand, the drug release rate of the low-dose implant was constant, where the concentration gradient did not affect the kinetics. In general, the rate constants of the $1000 \mu \mathrm{g}$ implants were all higher than the $500 \mu \mathrm{g}$, including $k_{0}, k_{1}$, and $k_{H}$, indicating the drug release rate of the higher "total" dose was faster than the lower dose.

Both $1000 \mu \mathrm{g}$ and $500 \mu \mathrm{g}$ followed Higuchi model with high $R^{2}$ values $\left(R^{2}=0.98\right)$, indicating that drug release followed Fickian diffusion. Higuchi model describes pseudosteady-state diffusion where drug concentration is constant with time throughout the polymer membrane because drug solubility in the membrane is lower than in the reservoir and drug slowly dissolves in the membrane.

Following the mathematical analysis for Fick's law diffusion for a reservoir cylinder, where drug molecules diffuse from a lumen through a vessel wall, ${ }^{18}$

$$
\frac{M_{t}}{M_{0}}=1-\exp \left[-\frac{\left(R_{i} H+R_{o} H+2 R_{i} R_{o}\right) D K t}{R_{i}^{2} H\left(R_{0}-R_{i}\right)}\right]
$$


the diffusion coefficients $(D)$ of MTX through the membrane were $D=4.2 \times 10^{-10} \mathrm{~cm}^{2} / \mathrm{s}$ and $1.9 \times 10^{-10} \mathrm{~cm}^{2} / \mathrm{s}$, for the $1000 \mu \mathrm{g}$ and $500 \mu \mathrm{g}$, respectively, where $R_{i}$ is inner diameter, $R_{o}$ is outer diameter, $H$ is the length of the cylinder (implant), and $K$ is the partition coefficient of MTX between the membrane and the reservoir. We assume $K=1$ and MTX is penetrating through the pores. We found that the effective diffusion coefficient of MTX through the nanopores was approximately 1000 times less than the diffusion coefficient of MTX in water. In addition, the $D$ was proportional to the rate constant of the first-order kinetics $k_{1}$ and the drug release rate of the $1000 \mu \mathrm{g}$ was twice as fast as the $500 \mu \mathrm{g}$. These results were consistent with other studies, which described that increasing the loading of therapeutic particles in the matrix also increases the release rate of therapeutics. ${ }^{19-22}$ Also the effective diffusion coefficients based on Fick's law were in the same order at $10^{-10} \mathrm{~cm}^{2} / \mathrm{s}$ and the values were increasing with the loading capacity.

In vivo drug release was successfully shown via the implant's fluorescence intensity changes (Figures 5 and 6). The mean gray values in the implant decreased while the value in the vitreous increased after laser irradiation. The laser used in this study was a pulsed picosecond laser with 700 ps pulse duration, $10 \mathrm{kHz}$ repetition frequency at $1064 \mathrm{~nm}$ wavelength at $1 \mathrm{~W}$. Although our previous study has shown that drug was released by a single pulse of this laser, ${ }^{23} 5 \mathrm{~s}$ was used per one spot in order to release physiologically relevant dose in this study. Our other previous study also has shown that the longer laser duration, the more drug is released. ${ }^{24}$ For the safety of the retina, we calculated maximum permissible exposure based on the following equation. 25

$$
P(W)=1.8 \times 10^{-3} C_{A} T^{0.75}\left(0.385 \mathrm{~cm}^{2}\right) F
$$

where $C_{A}=5$ for $(\lambda=1064 \mathrm{~nm}), T$ is total irradiation duration, $0.385 \mathrm{~cm}^{2}$ is area of 7 $\mathrm{mm}$ pupil, and $F$ is pulse repetition frequency. The calculation suggested that the laser intensity for $5 \mathrm{~s}$ at $1 \mathrm{~W}$ is 9 times higher than the maximum permissible exposure value with a focal length at $44 \mathrm{~mm}$. Note that this power is calculated when the laser directly hit the retina whereas laser should be targeted to implants. In the future studies, power as low as $100 \mathrm{~mW}$ will be used to avoid potential laser damage.

\section{Conclusion}

In this study, we successfully showed effective drug release from a nanoporous PLGA implant using pulsed NIR laser irradiation both in vitro and in vivo. We analyzed the drug release kinetics in vitro by fitting utilizing zero order, first order, and KP and Highchi models. We quantified multiple drug releases in the vitreous in the in vivo fluorescence study, consistent with the in vitro data. The dose released from the implant after the laser irradiation trigger was also clinically relevant. Histology and optical and ultrasound imaging data suggest the drug delivery system is not toxic to the retina. This drug delivery system could be potentially used for long-term posterior eye disease treatment. 


\section{Acknowledgment}

This study was partially supported by Ohio Lions Eye Research Foundation, Office of Research at University of Cincinnati, and NIH KL2 award (5KL2TR001426-04). The authors would like to thank Drs. Jonathon Nickels and Vesselin Shanov for equipment.

\section{References}

1. Lee, R.; Wong, T. Y.; Sabanayagam, C., Epidemiology of diabetic retinopathy, diabetic macular edema and related vision loss. Eye Vis (Lond) 2015, 2, 17-17.

2. Pennington, K. L.; DeAngelis, M. M., Epidemiology of age-related macular degeneration (AMD): associations with cardiovascular disease phenotypes and lipid factors. Eye Vis (Lond) 2016, 3, 34-34.

3. del Amo, E. M.; Rimpelä, A.-K.; Heikkinen, E.; Kari, O. K.; Ramsay, E.; Lajunen, T.; Schmitt, M.; Pelkonen, L.; Bhattacharya, M.; Richardson, D.; Subrizi, A.; Turunen, T.; Reinisalo, M.; Itkonen, J.; Toropainen, E.; Casteleijn, M.; Kidron, H.; Antopolsky, M.; Vellonen, K.-S.; Ruponen, M.; Urtti, A., Pharmacokinetic aspects of retinal drug delivery. Progress in Retinal and Eye Research 2017, 57, 134-185.

4. Kane, F. E.; Burdan, J.; Cutino, A.; Green, K. E., Iluvien ${ }^{\text {TM }}$ : a new sustained delivery technology for posterior eye disease. Expert Opinion on Drug Delivery 2008, 5 (9), 1039-1046.

5. Bezatis, A.; Spital, G.; Höhn, F.; Maier, M.; Clemens, C. R.; Wachtlin, J.; Lehmann, F.; Hattenbach, L. O.; Feltgen, N.; Meyer, C. H., Functional and anatomical results after a single intravitreal Ozurdex injection in retinal vein occlusion: a 6-month follow-up - The SOLO study. 2013, 91 (5), e340-e347.

6. Haghjou, N.; Soheilian, M.; Abdekhodaie, M. J., Sustained release intraocular drug delivery devices for treatment of uveitis. Journal of ophthalmic \& vision research 2011, 6 (4), 317-29.

7. Allergan, Ozurdex ten years 2019.

8. He, X.; Yuan, Z.; Kao, W.; Miller, D.; Li, S. K.; Park, Y. C., Size-Exclusive Nanoporous Biodegradable PLGA Capsules for Drug Delivery Implants and In Vivo Stability in the Posterior Segment. ACS Applied Bio Materials 2020, 3 (3), 1722-1729.

9. Rathinam, S. R.; Gonzales, J. A.; Thundikandy, R.; Kanakath, A.; Murugan, S. B.; Vedhanayaki, R.; Lim, L. L.; Suhler, E. B.; Al-Dhibi, H. A.; Doan, T.; Keenan, J. D.; Rao, M. M.; Ebert, C. D.; Nguyen, H. H.; Kim, E.; Porco, T. C.; Acharya, N. R., Effect of Corticosteroid-Sparing Treatment With Mycophenolate Mofetil vs Methotrexate on Inflammation in Patients With Uveitis: A Randomized Clinical Trial. Jama 2019, 322 (10), 936-945.

10. Korsmeyer, R. W.; Peppas, N. A., Effect of the morphology of hydrophilic polymeric matrices on the diffusion and release of water soluble drugs. Journal of Membrane Science 1981, 9 (3), 211-227.

11. Peppas, N. A., Analysis of Fickian and non-Fickian drug release from polymers. Pharmaceutica acta Helvetiae 1985, 60 (4), 110-1.

12. Higuchi, T., Mechanism of sustained-action medication. Theoretical analysis of rate of release of solid drugs dispersed in solid matrices. Journal of pharmaceutical sciences 1963, 52 (12), 1145-1149.

13. Thackaberry, E. A.; Farman, C.; Zhong, F.; Lorget, F.; Staflin, K.; Cercillieux, A.; Miller, P. E.; Schuetz, C.; Chang, D.; Famili, A., Evaluation of the toxicity of intravitreally injected PLGA microspheres and rods in monkeys and rabbits: effects of depot size on inflammatory response. Investigative ophthalmology \& visual science 2017, 58 (10), 4274-4285.

14. Schmitz-Valckenberg, S.; Guo, L.; Maass, A.; Cheung, W.; Vugler, A.; Moss, S. E.; Munro, P. M. G.; Fitzke, F. W.; Cordeiro, M. F., Real-Time In Vivo Imaging of Retinal Cell Apoptosis after Laser Exposure. Investigative ophthalmology \& visual science 2008, 49 (6), 2773-2780. 
15. Shulman, S.; Belokopytov, M.; Dubinsky, G.; Belkin, M.; Rosner, M., Ameliorative effect of PN277 on laser-induced retinal damage. Graefe's Archive for Clinical and Experimental Ophthalmology 2009, 247 (3), 343-348.

16. Benner, J. D.; Dao, D.; Butler, J. W.; Hamill, K. I., Intravitreal methotrexate for the treatment of proliferative vitreoretinopathy. BMJ Open Ophthalmology 2019, 4 (1), e000293.

17. Palakurthi, N. K.; Krishnamoorthy, M.; Augsburger, J. J.; Correa, Z. M.; Banerjee, R. K., Investigation of kinetics of methotrexate for therapeutic treatment of intraocular lymphoma. Current eye research 2010, 35 (12), 1105-15.

18. Siepmann, J.; Siepmann, F., Modeling of diffusion controlled drug delivery. Journal of Controlled Release 2012, 161 (2), 351-362.

19. Saltzman, W. M.; Langer, R., Transport rates of proteins in porous materials with known microgeometry. Biophys. J. 1989, 55 (1), 163-171.

20. Siegel, R. A.; Langer, R., Controlled release of polypeptides and other macromolecules. Pharmaceutical research 1984, 1 (1), 2-10.

21. Miller, E. S.; Peppas, N. A., DIFFUSIONAL RELEASE OF WATER-SOLUBLE BIOACTIVE AGENTS FROM ETHYLENE-VINYL ACETATE COPOLYMERS. Chemical Engineering Communications 1983, 22 (5-6), 303-315.

22. Rhine, W. D.; Hsieh, D. S. T.; Langer, R., Polymers for sustained macromolecule release: Procedures to fabricate reproducible delivery systems and control release kinetics. Journal of pharmaceutical sciences 1980, 69 (3), 265-270.

23. Zhang, Z.; Taylor, M.; Kaval, N.; Park, Y. C., Phase-Transition Temperature of Gold-NanorodCoated Nanodroplets to Microbubbles by Pulsed Laser. The Journal of Physical Chemistry A 2019, 123 (23), 4844-4852.

24. Das, S.; Lazenby, R. A.; Yuan, Z.; White, R. J.; Park, Y. C., Effect of Laser Irradiation on Reversibility and Drug Release of Light-Activatable Drug-Encapsulated Liposomes. Langmuir 2020.

25. Delori, F. C.; Webb, R. H.; Sliney, D. H., Maximum permissible exposures for ocular safety (ANSI 2000), with emphasis on ophthalmic devices. J. Opt. Soc. Am. A 2007, 24 (5), 1250-1265. 